\title{
Geometric Quantization of Symplectic Manifolds with Respect to Reducible Non-Negative Polarizations
}

\author{
Jørgen Ellegaard Andersen \\ Department of Mathematics, University of Aarhus, DK-8000 Aarhus, Denmark. \\ E-mail: andersen@mi.aau.dk
}

Received: 16 November 1995/Accepted: 2 July 1996

\begin{abstract}
The leafwise complex of a reducible non-negative polarization with values in the prequantum bundle on a prequantizable symplectic manifold is studied. The cohomology groups of this complex is shown to vanish in rank less than the rank of the real part of the non-negative polarization. The Bohr-Sommerfeld set for a reducible non-negative polarization is defined. A factorization theorem is proved for these reducible non-negative polarizations. For compact symplectic manifolds, it is shown that the above complex has finite dimensional cohomology groups, moreover a Lefschetz fixed point theorem and an index theorem for these non-elliptic complexes is proved. As a corollary of the index theorem, we deduce that the cardinality of the Bohr-Sommerfeld set for any reducible real polarization on a compact symplectic manifold is determined by the volume and the dimension of the manifold.
\end{abstract}

\section{Contents}

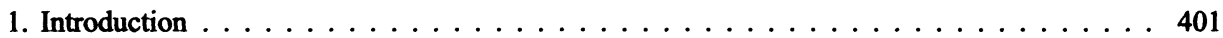

2. The leafwise complex of a non-negative reducible polarization . . . . . . . . 403

3. A Lefschetz fixed point theorem for reducible non-negative polarizations . . . . . . . . 413

4. An index theorem for reducible non-negative polarizations $\ldots \ldots \ldots \ldots \ldots \ldots$

\section{Introduction}

Let $M$ be a symplectic manifold. Assume that $M$ is prequantizable, that is there exists a complex Hermitian line bundle $\mathscr{L}$ over $M$ with a compatible connection $\nabla$, whose curvature is the symplectic form on $M$. In the program of geometric quantization one is asked to consider a polarization $P$ on $M$ and to study the cohomology groups of the leafwise complex with respect to $P$ with values in $\mathscr{L}$. See e.g. $[5,6,8]$. Traditionally, Kähler polarizations have received most attention, since the

Supported in part by NSF grant DMS-93-09653, while the author was visiting University of California Berkeley. 
powerful theory of elliptic operators between Hilbert spaces is applicable in this case, and one gets the machinery of Hodge theory and the index and Lefschetz theorems. In this paper we shall try to initiate the study of index and Lefschetz fixed point theory for more general polarizations. The natural complex with values in the prequantum line bundle $\mathscr{L}$ on the symplectic manifold $M$ with a non-negative polarization $P$ is:

$$
\begin{gathered}
0 \rightarrow C^{\infty}\left(\mathscr{L}^{k} \otimes \delta_{P}\right) \stackrel{\nabla^{P}}{\rightarrow} C^{\infty}\left(\bar{P}^{*} \otimes \mathscr{L}^{k} \otimes \delta_{P}\right) \\
\stackrel{\nabla^{P}}{\rightarrow} \cdots \stackrel{\nabla^{P}}{\rightarrow} C^{\infty}\left(\Lambda^{n} \bar{P}^{*} \otimes \mathscr{L}^{k} \otimes \delta_{P}\right) \rightarrow 0
\end{gathered}
$$

which is non-elliptic, if $P$ is not a Kähler polarization (here $\delta_{P}$ is the normal halfform bundle of $P$ ). The study of this complex goes back to the initial study of geometric quantization. See e.g. [5].

We prove in this paper, using a spectral sequence argument, that if the polarization is reducible (meaning the leaf spaces $M /(P+\bar{P})$ and $M /(P \cap \bar{P})$ are Hausdorff manifolds and the leaves of the distribution $P \cap \bar{P}$ are compact), then some kind of a factorization result holds. It says that

$$
H^{i}\left(M, \nabla^{P}\right)=0, \quad 0 \leqq i<m,
$$

where $m$ is the number of real directions in $P$, i.e. $\operatorname{rk}(P \cap \bar{P})=m$, and

$$
H^{i}\left(M, \nabla^{P}\right)=\prod_{b \in S_{k}} H^{i-m}\left(M_{b}^{\prime}, \bar{\partial}_{b}\right), \quad m \leqq i \leqq n .
$$

Here $S_{k}$ is the Bohr-Sommerfeld subset of the leaf space $M /(P+\bar{P})$, and $M_{b}^{\prime}$ is the symplectic reduction at $b$. (See Sect. 2 for further explanation of the notation.) If $M$ is compact, we see in particular from this, that the cohomology groups $H^{i}\left(M, \nabla^{P}\right)$ are finite dimensional.

Now suppose we have a symplectomorphism $f$ with simple fixed points of $M$ that preserves $P$, and assume moreover we have a lift $f^{*}$ of $f$ to $\mathscr{L}$. Assume moreover that $M$ is compact. A local analysis of $f$ combines, by the use of the spectral sequence, with the holomorphic Lefschetz fixed point theorem to prove a Lefschetz fixed point theorem for the complex (1):

$$
\begin{aligned}
\sum_{i}(-1)^{i} \operatorname{Tr}\left(f^{*}: H^{i}\left(M, \nabla^{P}\right)\right) \\
=\sum_{\{p \in M \mid f(p)=p\}} v_{p} \sum_{i}(-1)^{i} \frac{\operatorname{Tr}\left(f_{p}^{*}: \Lambda^{i} \bar{P}_{p}^{*} \otimes \mathscr{L}_{p}^{k} \otimes \delta_{P_{p}}\right)}{|\operatorname{Det}(1-d f(p))|},
\end{aligned}
$$

where

$$
v_{p}=\operatorname{Sign} \operatorname{Det}\left(1-d f_{B}(p)\right),
$$

and $f_{B}$ is the induced map on the leaf space $M /(P+\bar{P})$.

We can also prove an index theorem for these non-elliptic complexes under a somewhat technical assumption on the non-negative polarization. This assumption is trivially satisfied for real polarizations and Kähler polarizations. The proof of this index theorem is by a combination of a topological argument and the use of the Hirzebruch-Riemann-Roch formula applied to the Kähler manifolds $M_{b}^{\prime}, b \in S_{k}$. This index theorem says that

$$
\operatorname{Index}\left(\nabla^{P}\right)=(-1)^{m} \int_{M} \operatorname{ch}\left(\mathscr{L}^{k}\right) \wedge \widehat{A}(T M) .
$$


Here $\operatorname{Index}\left(\nabla^{P}\right)$ is the index of the complex (1), i.e. the alternating sum of the dimensions of the cohomology groups $H^{i}\left(M, \nabla^{P}\right)$.

When one applies this index theorem to a reducible real polarization, one gets the result that the volume and the dimension of $M$ determines the cardinality of the level $k$ Bohr-Sommerfeld set $S_{k}$ of $P$ :

$$
\# S_{k}=k^{n} \operatorname{Vol}(\mathrm{M}) \text {. }
$$

We shall end this introduction by giving some examples of symplectic manifolds which are non-Kähler, but which do admit reducible real polarizations. Let $\widetilde{S} L(2, \mathbb{Z})$ be the semi-direct product of $S L(2, \mathbb{Z})$ and $\mathbb{R}^{2}$. Consider the representation

$$
\rho: \mathbb{Z}^{2} \rightarrow \tilde{S} L(2, \mathbb{Z}),
$$

given by

$$
\begin{gathered}
h(1,0)(x, y)=(x+1, y), \\
h(0,1)(x, y)=(x+y, y+1) .
\end{gathered}
$$

The quotient $\mathbb{R}^{2} / \rho\left(\mathbb{Z}^{2}\right)$ is the 2-torus $T^{2}$ with its non-standard $\tilde{S} L(2, \mathbb{Z})$ structure, thought of as an affine manifold. There is a naturally defined lagrangian submanifold $\Lambda$ of $T^{*} T^{2}$ defined by requiring that

$$
\Lambda_{p}=\Lambda \cap T_{p}^{*} T^{2} \subset T_{p}^{*} T^{2}
$$

is identified with $\mathbb{Z}^{2} \subset \mathbb{R}^{2}$ in affine $\tilde{S} L(2, \mathbb{Z})$-coordinates for all $p \in T^{2}$. The quotient $M=T_{p}^{*} T^{2} / \Lambda$ is a compact symplectic manifold and the vertical polarization of $T_{p}^{*} T^{2}$ induces a reducible real polarization of $M$. One notices that any affine $\tilde{S} L(n, \mathbb{Z})$ manifold in the above way gives rise to a compact symplectic manifold of dimension $2 n$ which admits a reducible real polarization.

In the particular case at hand, we notice that the four dimensional manifold $M$ admits no Kähler structures. It is not even homotopy equivalent to a Kähler manifold, because the first Betti-number is 3. In fact $M$ is diffeomorphic to Thurston's first example of a non-Kähler compact symplectic manifold (see [7], p. 10). Notice moreover that Thurston's example also supports a reducible real polarization, which in the notation of [7] is simply given by projecting onto the $p_{i}^{\prime} s$.

The author wishes to thank Prof. J. Roe and Prof. G. Segal for stimulating discussions on this project.

\section{The Leafwise Complex of a Non-Negative Reducible Polarization}

In this section we shall study the differential geometry and the local structure of smooth polarizations on symplectic manifolds. This study will enable us to describe the cohomology groups of the leafwise complex with values in the prequantum bundle, associated to certain kinds of polarizations.

Let us first briefly review the complex Lagrangian Grassmannian of a symplectic vector space. Let $(V, \omega)$ be a real $2 n$-dimensional symplectic vector space. Extend $\omega$ complex linearly to $V \otimes \mathbb{C}$. Consider the complex Lagrangian Grassmannian $L(V \otimes \mathbb{C})$, the space of non-negative Lagrangian subspaces of $V \otimes \mathbb{C}$. Recall, that a subspace $W$ is non-negative if

$$
-i \omega(v, \bar{v}) \geqq 0 \quad \forall v \in W .
$$


Note that non-negativity is part of our definition of a Lagrangian subspace, since we will only be interested in such subspaces. There is a function

$$
\rho: L(V \otimes \mathbb{C}) \rightarrow\{0, \ldots, n\},
$$

which counts the number of real directions in a subspace, i.e.

$$
\rho(W)=\operatorname{dim}(W \cap \bar{W} \cap V), \quad W \in L(V \otimes \mathbb{C}) .
$$

We use the following notation:

$$
L_{i}(V \otimes \mathbb{C})=\rho^{-1}(i)
$$

General theory (see [8]) shows that $L(V \otimes \mathbb{C})$ is topologically a closed ball. The interior of $L(V \otimes \mathbb{C})$ is $L_{0}(V \otimes \mathbb{C})$ and

$$
\overline{L_{k}(V \otimes \mathbb{C})}=\bigcup_{m=k}^{n} L_{m}(V \otimes \mathbb{C}) .
$$

Suppose now $\left(M^{2 n}, \omega\right)$ is a symplectic manifold. Denote by $L(M)$ the topological fibre bundle over $M$, whose fibre over $p \in M$ is $L_{p}(M)=L\left(T_{p} M \otimes \mathbb{C}\right)$. Now assume $P: M \rightarrow L(M)$ is a section of this fibration. Define

$$
U_{l}(P)=\{p \in M \mid \rho(P(p))=l\} .
$$

These sets give a partition of $M$ for each section $P$ :

$$
M=\bigcup_{l=0}^{n} U_{l}(P)
$$

We now make the definition of a non-negative polarization.

Definition 2.1. A non-negative polarization of a symplectic manifold $(M, \omega)$ is a section $P: M \rightarrow L(M)$ with the properties that $P$ is a smooth subbundle of $T M \otimes \mathbb{C}$, and $P$ is integrable, meaning that around each point $p \in M$ there are $n$ smooth complex functions, whose Hamiltonian vector fields trivialize $P$ in $a$ neighbourhood of $p$.

Notice that $\rho(P(p))$ is required to be constant over $M$. One could allow more general polarizations where $\rho(P(p))$ is allowed to vary. Such will not be considered here.

On a symplectic manifold one has a natural way to differentiate vector fields in a coisotropic foliation along the isotropic directions. Since a polarization is both isotropic and coisotropic, we can differentiate sections of $\bar{P}$ along directions in $\bar{P}$, by defining

$$
\tilde{\nabla}: C^{\infty}(M, \bar{P}) \rightarrow C^{\infty}\left(M, \bar{P}^{*} \otimes \bar{P}\right)
$$

according to

$$
i_{\left(\tilde{\nabla}_{X} Y\right)} \omega=i_{X} d\left(i_{Y} \omega\right) \quad \forall X, Y \in C^{\infty}(M, \bar{P}) .
$$

We say that $\tilde{\nabla}$ is a $\bar{P}$-connection in $\bar{P}$. Now $\tilde{\nabla}$ induces a $\bar{P}$-connection in $\Lambda^{n} \bar{P}^{*}$.

Assume a "normal half-form" bundle $\delta_{P}$ for the polarization $P$ has been chosen. By definition (see e.g. [8]) a normal half-form bundle for $P$ is a complex line bundle 
with a $\bar{P}$ connection, such that

$$
\Lambda^{n} \bar{P}^{*} \cong \delta_{P} \otimes \delta_{P}
$$

as complex line bundles with $\bar{P}$-connection.

Suppose $(\mathscr{L}, \nabla)$ is a prequantum line bundle over $M$. That means $\mathscr{L}$ is a Hermitian line bundle with a compatible connection $\nabla$, such that the curvature two form of $\nabla$ is $\omega$. Recall (see e.g. [8], Prop. (8.3.1)) that the prequantum line bundle exists if and only if $[\omega] \in H^{2}(M, \mathbb{R})$ is contained in the image of $H^{2}(M, \mathbb{Z})$ in $H^{2}(M, \mathbb{R})$. Moreover, the inequivalent choices of the prequantum line bundle are parametrized by $H^{1}(M, U(1))$, when this condition is satisfied. By restricting the connection in $\mathscr{L}$, we get a $\bar{P}$-connection in the bundle $\mathscr{L}^{k} \otimes \delta_{P}$. We are interested in studying the leafwise complex:

$$
0 \rightarrow C^{\infty}\left(\mathscr{L}^{k} \otimes \delta_{P}\right) \stackrel{\nabla^{P}}{\rightarrow} C^{\infty}\left(\bar{P}^{*} \otimes \mathscr{L}^{k} \otimes \delta_{P}\right) \stackrel{\nabla^{P}}{\rightarrow} \cdots \stackrel{\nabla^{P}}{\rightarrow} C^{\infty}\left(\Lambda^{n} \bar{P}^{*} \otimes \mathscr{L}^{k} \otimes \delta_{P}\right) \rightarrow 0 .
$$

We shall describe the cohomology groups of this complex for "special" polarizations. Let us now specify the kinds of polarizations on which we will concentrate.

Consider the two distributions

$$
E=(P+\bar{P}) \cap T M
$$

and

$$
D=P \cap \bar{P} \cap T M .
$$

By the definition of a polarization both $D$ and $E$ have constant rank, say $m$ and $2 n-m$ respectively.

Definition 2.2. We say a polarization is reducible if $E$ and $D$ are integrable and reducible, meaning that the leafspaces $B=M / E$ and $M^{\prime}=M / D$ are Hausdorff manifolds and the leaves of $D$ are compact.

In the rest of this chapter, we shall only consider reducible polarizations. For such polarizations we have the locally trivial projections

$$
\pi_{E}: M \rightarrow B
$$

and

$$
\pi_{D}: M \rightarrow M^{\prime}
$$

Furthermore we have a projection

$$
\pi_{B}: M^{\prime} \rightarrow B
$$

which is a locally trivial fibration, such that

$$
\pi_{E}=\pi_{B} \circ \pi_{D} .
$$

The symplectic form gives us an isomorphism

$$
\omega: T_{p} M \rightarrow T_{p}^{*} M,
$$

and so we get an inclusion

$$
T_{\pi_{E}(p)}^{*} \stackrel{\pi_{E}^{*}}{\longrightarrow} T_{p}^{*} M \stackrel{\omega^{-1}}{\rightarrow} T_{p} M,
$$


the image being $D_{p}$. Hence we see that $T_{b}^{*} B$ acts infinitesimally on $\pi_{E}^{-1}(b)$. But since $\pi_{D}^{-1}\left(p^{\prime}\right) \subset \pi_{E}^{-1}(b)$ is compact for all $p^{\prime} \in \pi_{E}^{-1}(b)$ and $T_{b}^{*} B$ is abelian, the infinitesimal action exponentiates to an action of $T_{b}^{*} B$ on $\pi_{E}^{-1}(b)$. Let us denote $\pi_{E}^{-1}(b) / D=\pi_{D}\left(\pi_{E}^{-1}(b)\right)$ by $M_{b}^{\prime}$. Note that the polarization $P$ induces a Kähler structure on $M_{b}^{\prime}$. Let $p^{\prime} \in M_{b}^{\prime}$, then $T_{b}^{*} B$ acts locally transitively, and therefore transitively on $\pi_{D}^{-1}\left(p^{\prime}\right)$. (See p. 351 in [3].) This means that

$$
\Lambda_{b, p^{\prime}}=\left\{\alpha \in T_{b}^{*} B|\alpha|_{\pi_{D}^{-1}\left(p^{\prime}\right)}=\mathrm{Id}\right\}
$$

is a discrete lattice of maximal $\operatorname{rank}$ in $T_{b}^{*} B$, and we have an identification

$$
\pi_{D}^{-1}\left(p^{\prime}\right) \cong \frac{T_{b}^{*} B}{\Lambda_{b, p^{\prime}}} \text {. }
$$

Hence $\pi_{D}: \pi_{E}^{-1}(b) \rightarrow M_{b}^{\prime}$ is a $T^{m}$ fibre bundle.

We note that when we restrict the bundle $\mathscr{L}^{k} \otimes \delta_{P}$ to $\pi_{D}^{-1}\left(p^{\prime}\right)$, we get a flat line bundle on the torus $\pi_{D}^{-1}\left(p^{\prime}\right)$, i.e. an element of the dual torus of $\pi_{D}^{-1}\left(p^{\prime}\right)$. When we say that $\left.\mathscr{L}^{k} \otimes \delta_{P}\right|_{\pi_{D}^{-1}\left(p^{\prime}\right)}$ is trivial, we mean trivial as a flat line bundle with connection.

Definition 2.3. The level $k$ Bohr-Sommerfeld set for $P$ is defined to be

$$
S_{k}=\left\{b \in B\left|\left(\mathscr{L}^{k} \otimes \delta_{P}\right)\right|_{\pi_{D}^{-1}\left(p^{\prime}\right)} \text { is trivial for all } p^{\prime} \in M_{b}^{\prime}\right\} .
$$

In fact, if there is a $p^{\prime} \in M_{b}^{\prime}$ such that $\left.\left(\mathscr{L}^{k} \otimes \delta\right)\right|_{\pi_{D}^{-1}\left(p^{\prime}\right)}$ is trivial, then $b \in S_{k}$. This follows from the fact that the symplectic annihilator of $E$ is $D$. Moreover, we will see later in this section that $S_{k}$ is a union of isolated points on $B$. This definition is a straightforward generalization of the classical definition of the Bohr-Sommerfeld set for a reducible real polarization (see [8], pp. 201, 241 and 251-53).

Suppose $b \in S_{k}$. By the definition of $S_{k}$, there will exist a holomorphic line bundle over $M_{b}^{\prime}$, which we will denote $\left(\mathscr{L}_{b}^{k} \otimes K_{b}^{\frac{1}{2}}, \bar{\partial}_{b}\right)$, such that $\left(\pi_{D}^{*}\left(\mathscr{L}_{b}^{k} \otimes K_{b}^{\frac{1}{2}}\right), \pi_{D}^{*} \bar{\partial}_{b}\right)$ is isomorphic to $\left(\left.\left(\mathscr{L}^{k} \otimes \delta_{P}\right)\right|_{\pi_{D}^{-1}(b)}, \nabla^{P}\right)$. We have that

$$
\begin{aligned}
\pi_{D}^{*}\left(\left(\mathscr{L}^{k} \otimes K_{b}^{\frac{1}{2}}\right) \otimes\left(\mathscr{L}^{k} \otimes K_{b}^{\frac{1}{2}}\right)\right) & \left.\cong \mathscr{L}^{2 k}\right|_{\pi_{D}^{-1}(b)} \otimes \Lambda^{n} \bar{P}^{*} \\
& \left.\cong \mathscr{L}^{2 k}\right|_{\pi_{D}^{-1}(b)} \otimes \pi_{D}^{*} K_{b} .
\end{aligned}
$$

Here $K_{b}$ is the canonical bundle of $M_{b}^{\prime}$. This explains our notation $\left(\mathscr{L}_{b}^{k} \otimes K_{b}^{\frac{1}{2}}, \bar{\partial}_{b}\right)$. (Note that $\mathscr{L}_{b}^{k}$ and $K_{b}^{\frac{1}{2}}$ may not exist, but their tensor product $\mathscr{L}_{b}^{k} \otimes K_{b}^{\frac{1}{2}}$ will exist.)

Locally we have the following identifications. Let $b \in B$, then there is an open neighbourhood $U$ of $b$, over which the fibrations are trivial, i.e.

$$
\pi_{E}^{-1}(U) \cong \pi_{E}^{-1}(b) \times U
$$

and

$$
\pi_{B}^{-1}(U) \cong M_{b}^{\prime} \times U
$$


Moreover, for each $p=\left(p^{\prime}, b^{\prime}\right) \in \pi_{B}^{-1}(U)$ there is a neighbourhood $V$ of $p^{\prime} \in M_{b}^{\prime}$ such that

$$
\pi_{D}^{-1}(V \times U) \cong T^{m} \times V \times U
$$

We will denote $\pi_{D}^{-1}(V \times U)=W$.

These trivializations show we can find a smooth section

$$
\tilde{s}: V \times U \rightarrow W
$$

of $\pi_{D}: W \rightarrow V \times U$. It is clear that $\tilde{s}_{v}^{*} \omega, v \in V$ is a smooth family of closed forms on $U$. Hence we can find a smooth family $\alpha_{v}, v \in V$ of 1-forms, such that

$$
-d_{U} \alpha_{v}=\tilde{s}_{v}^{*} \omega .
$$

Now $\alpha_{v}$ is a section of $T^{*} U$, and therefore by the fiberwise action gives a diffeomorphism

$$
\kappa_{\alpha_{v}}: T^{m} \times\{v\} \times U \hookleftarrow .
$$

We define a new section $s_{v}: U \rightarrow W, v \in V$, by

$$
s_{v}=\kappa_{\alpha_{v}} \tilde{s_{v}} \text {. }
$$

We then have that

$$
s_{v}^{*} \omega=0, \quad v \in V .
$$

For each $v \in V$ we get a map

$$
\chi_{0}: T^{*} U \rightarrow W
$$

given by

$$
\chi_{v}(\xi)=-\xi s_{v}(x)
$$

We have the following

Proposition 2.1. $\chi_{v}: T^{*} U \rightarrow M$ is locally symplectic with respect to the standard symplectic structure on $T^{*} U$, i.e. $\chi_{v}^{*} \omega$ is the standard symplectic structure on $T^{*} U$.

Proof. The proof is exactly the same as the proof of Theorem 44.2 in [3], pp. 354-355.

From this proposition it follows that we can therefore find smooth functions $x_{i}: V \times U \rightarrow \mathbb{R}$, such that $\left(x_{1}^{v}, \ldots, x_{m}^{v}\right)$ form coordinates on $U$ for all $v \in V$, and $d x_{1}^{v}, \ldots, d x_{m}^{v}$ form a basis of $\Lambda_{U, v}$. There are uniquely defined smooth functions $y_{1}, \ldots, y_{m}: W \rightarrow \mathbb{R} / \mathbb{Z}$, such that $\left(x_{1}^{v}, \ldots, x_{m}^{v}, \chi_{v}^{*}\left(y_{1}\right), \ldots, \chi_{v}^{*}\left(y_{m}\right)\right)$ are canonical symplectic coordinates on $T^{*} U / \Lambda_{U, v}$. Define a 1 -form $\alpha^{\prime}$ on $W$ by

$$
\alpha^{\prime}=\sum_{i=1}^{m} x_{i} d y_{i} .
$$

It is then clear that for any $v \in V$, we have that

$$
\chi_{v}^{*}\left(\omega-d \alpha^{\prime}\right)=0 .
$$

The 1 -form $\alpha^{\prime}$ defines a connection in the trivial complex line bundle over $W$. Let us denote this bundle with connection $\mathscr{L}^{\prime}$. 
The restriction of $\mathscr{L}$ to $W$ is topologically trivial, so there will be a non-zero section $\lambda$ of $\mathscr{L}$ over $W$. Let $\alpha$ be the connection 1-form defined by $\lambda$ :

$$
\nabla \lambda=i \alpha \otimes \lambda \text {. }
$$

Then $d \alpha=\omega$. For $v \in V$ let us normalize the coordinates $x^{v}$, such that

$$
\left[\left.\alpha\right|_{\pi_{D}^{-1}(v, b)}\right]=\left[\left.\alpha^{\prime}\right|_{\pi_{D}^{-1}(v, b)}\right]
$$

as elements of $H^{1}\left(\pi_{D}^{-1}(v, b), \mathbb{R}\right)$.

Proposition 2.2. With the given normalization of the coordinates $x$, we have that $\left.\mathscr{L}\right|_{W} \otimes\left(\mathscr{L}^{\prime}\right)^{*}$ is trivial as a bundle with connection along the fibres of $D$ over $V \times U$.

Proof. When we restrict to the image of $\chi_{v}$, which is $\pi_{D}^{-1}(\{v\} \times U)$, we see that

$$
d\left(\left.\alpha\right|_{\pi_{D}^{-1}(\{v\} \times U)}-\left.\alpha^{\prime}\right|_{\pi_{D}^{-1}(\{v\} \times U)}\right)=\left.\omega\right|_{\pi_{D}^{-1}(\{v\} \times U)}-\left.d \alpha^{\prime}\right|_{\pi_{D}^{-1}(\{v\} \times U)}=0 .
$$

By the normalization of $x$ we have that

$$
\left[\left.\alpha\right|_{\pi_{D}^{-1}(\{v\} \times U)}-\left.\alpha^{\prime}\right|_{\pi_{D}^{-1}(\{v\} \times U)}\right] \in H^{1}\left(\pi_{D}^{-1}(\{v\} \times U)\right)
$$

is zero. From this we conclude that $\left.\mathscr{L} \otimes\left(\mathscr{L}^{\prime}\right)^{*}\right|_{\pi_{D}^{-1}(\{v\} \times U)}$ is trivial.

For $b^{\prime} \in U$ consider the bundle $\left.\mathscr{L} \otimes\left(\mathscr{L}^{\prime}\right)^{*}\right|_{\pi_{E}^{-1}\left(b^{\prime}\right) \cap W}$. Since this bundle is trivial along the fibres of $\pi_{D}$, we see that there exists a prequantum bundle $\mathscr{L}_{b^{\prime}}$ over $V \times\left\{b^{\prime}\right\}$, such that

$$
\left.\mathscr{L}\right|_{\pi_{E}^{-1}\left(b^{\prime}\right) \cap W} \cong \mathscr{L}^{\prime} \otimes \pi_{D}^{*}\left(\mathscr{L}_{b^{\prime}}\right) \text {. }
$$

From this, it follows that we can find complex functions $z=\left(z_{m+1}, \ldots, z_{n}\right)$ on $V \times U$, such that

$$
\bar{P}=\operatorname{Span}\left\{\frac{\partial}{\partial y_{1}}, \ldots, \frac{\partial}{\partial y_{m}}, \frac{\partial}{\partial \bar{z}_{m+1}}, \ldots, \frac{\partial}{\partial \bar{z}_{n}}\right\},
$$

and a smooth complex function

$$
K: V \times U \rightarrow \mathbb{C},
$$

such that

$$
\tilde{\alpha}=\sum_{i=1}^{m} x_{i} d y_{i}+\sum_{i=m+1}^{n} \frac{\partial K}{\partial \bar{z}_{i}} d \bar{z}_{i}
$$

is a connection form for $\left.\mathscr{L}\right|_{\pi_{E}^{-1}\left(b^{\prime}\right) \cap W}, x=x\left(b^{\prime}\right)$. This and Proposition 2.1 imply in particular that there exists smooth functions $A_{i j}, B_{i j}: W \rightarrow \mathbb{C}$, such that

$$
\begin{aligned}
\omega= & \sum_{i=1}^{m} d x_{i} \wedge d y_{i}+\sum_{i=m+1}^{n}\left(A_{i j}+\frac{\partial^{2} K}{\partial x_{i} \partial \bar{z}_{j}}\right) d x_{i} \wedge d \bar{z}_{j} \\
& +\sum_{i=m+1}^{n} B_{i j} d x_{i} \wedge d z_{j}+\sum_{i=m+1}^{n} \frac{\partial^{2} K}{\partial z_{i} \partial \bar{z}_{j}} d z_{i} \wedge d \bar{z}_{j} .
\end{aligned}
$$


Since $\omega$ is closed, we must have that

$$
\frac{\partial A_{i j}}{\partial y_{k}}=0
$$

For any $\left(v, b^{\prime}\right) \in V \times U$ we can consider the flat bundle

$$
\left.\left(\Lambda^{n} \bar{P}\right)^{*}\right|_{\pi_{D}^{-1}\left(v, b^{\prime}\right)} \text {. }
$$

By using the explicit expression for $\omega$, one easily verifies that

$$
\tilde{\nabla}_{\frac{\partial}{\partial y_{i}}}\left(\frac{\partial}{\partial y_{1}} \wedge \cdots \wedge \frac{\partial}{\partial y_{m}} \wedge \frac{\partial}{\partial \bar{z}_{m+1}} \wedge \cdots \wedge \frac{\partial}{\partial \bar{z}_{n}}\right)=0 .
$$

Hence $\left.\left(\Lambda^{n} \bar{P}\right)^{*}\right|_{\pi_{D}^{-1}\left(v, b^{\prime}\right)}$ is trivial for all $\left(v, b^{\prime}\right) \in V \times U$. This means that the isomorphism class of $\left.\delta_{P}\right|_{\pi_{D}^{-1}\left(v, b^{\prime}\right)},\left(v, b^{\prime}\right) \in V \times U$ must be constant, since $\left.\left(\Lambda^{n} \bar{P}\right)^{*}\right|_{\pi_{D}^{-1}\left(v, b^{\prime}\right)}$ is trivial. That means that there exist constants $x_{i}^{0}, i=1, \ldots, m$, such that

$$
\alpha^{\prime \prime}=\sum_{i=1}^{m} x_{i}^{0} d y_{i}
$$

defines a connection in the trivial bundle, say $\delta^{\prime}$, over $W$, such that

$$
\left.\delta_{P} \otimes\left(\delta^{\prime}\right)^{*}\right|_{\pi_{D}^{-1}\left(v, b^{\prime}\right)}, \quad\left(v, b^{\prime}\right) \in V \times U
$$

is trivial, hence there exists $\delta_{b^{\prime}}$ a complex line bundle over $V \times\left\{b^{\prime}\right\}$, such that

$$
\left.\delta_{P}\right|_{\pi_{E}^{-1}\left(b^{\prime}\right) \cap W} \cong \delta^{\prime} \otimes \pi_{D}^{*} \delta_{b^{\prime}}
$$

Note that $\delta^{\prime} \otimes \delta^{\prime}$ is by construction trivial. We then have that

$$
\begin{aligned}
\pi_{D}^{*}\left(\delta_{b^{\prime}} \otimes \delta_{b^{\prime}}\right) & \left.\left.\cong\left(\delta_{P} \otimes \delta_{P}\right)\right|_{\pi_{E}^{-1}\left(b^{\prime}\right) \cap W} \otimes\left(\delta^{\prime} \otimes \delta^{\prime}\right)^{*}\right|_{\pi_{E}^{-1}\left(b^{\prime}\right) \cap W} \\
& \cong \Lambda^{n} \bar{P}^{*} \\
& \cong\left(\Lambda^{m} D\right)^{*} \otimes \pi_{D}^{*}\left(\left.K_{b^{\prime}}\right|_{V}\right) \\
& \cong \pi_{D}^{*}\left(\left.K_{b^{\prime}}\right|_{V}\right)
\end{aligned}
$$

Here $K_{b^{\prime}}$ is the canonical bundle of $M_{b^{\prime}}^{\prime}$. From this we conclude that $\delta_{b^{\prime}}$ defines a square root of $K_{b^{\prime}}$ over $V \times\left\{b^{\prime}\right\}$. We shall use the notation $K_{b^{\prime}}^{\frac{1}{2}}=\delta_{b^{\prime}}$.

Consider now the bundle $\mathscr{L} \otimes \delta_{P}$ with its $\bar{P}$ connection $\nabla^{P}$. We then have that

$$
\left.\left.\left(\mathscr{L} \otimes \delta_{P}\right)\right|_{\pi_{E}^{-1}\left(b^{\prime}\right) \cap W} \cong\left(\mathscr{L}^{\prime} \otimes \delta^{\prime}\right)\right|_{\pi_{E}^{-1}\left(b^{\prime}\right) \cap W} \otimes \pi_{D}^{*}\left(\mathscr{L}_{b^{\prime}}^{\prime} \otimes K_{b^{\prime}}^{\frac{1}{2}}\right)
$$

and

$$
\nabla^{P}=d_{D}+i \sum_{i=1}^{m} x_{i} d y_{i}+\pi_{D}^{*}\left(\bar{\partial}_{b^{\prime}}\right) .
$$

We have here changed the coordinates $\left(x_{i}\right)$ to $\left(x_{i}+x_{i}^{0}\right)$, and $\bar{\partial}_{b^{\prime}}$ is the $\bar{\partial}$-operator in $\mathscr{L}_{b^{\prime}}^{\prime} \otimes K_{b^{\prime}}^{\frac{1}{2}}$ over $V \times\left\{b^{\prime}\right\}$. We will use the notation

$$
\beta=\sum_{i=1}^{m} x_{i} d y_{i}
$$


It is clear from the expression for $\beta$ that $S_{k}$ is a discrete subset of $B$.

On the basis of this local analysis we can now prove the following factorization property.

Theorem 2.1. Suppose $M$ is a symplectic manifold and $(\mathscr{L}, \nabla)$ is a prequantum line bundle over $M$. For any reducible non-negative polarization $P$ on $M$, we have that

$$
H^{i}\left(M, \nabla^{P}\right)=0,
$$

for $0 \leqq i<m$, and there is a natural isomorphism

$$
\left(\pi_{D}\right)_{*}: H^{i}\left(M, \nabla^{P}\right) \rightarrow \prod_{b \in S_{k}} H^{i-m}\left(M_{b}^{\prime}, \bar{\partial}_{b}\right),
$$

for $m \leqq i \leqq n$.

Since $\mathscr{L}^{k} \otimes \delta_{P}$ is trivial along the fibres of $\pi_{D}: \pi_{E}^{-1}(b) \rightarrow M_{b}^{\prime}$ for $b \in S_{k}$, we get a well defined map

$$
\left(\pi_{D}\right)_{*}: C^{\infty}\left(M, \Lambda^{i} \bar{P}^{*} \otimes \mathscr{L}^{k} \otimes \delta_{P}\right) \rightarrow \prod_{b \in S_{k}} C^{\infty}\left(M_{b}^{\prime}, \Lambda^{0, i-m}\left(M_{b}^{\prime}\right) \otimes \mathscr{L}_{b}^{k} \otimes K_{b}^{\frac{1}{2}}\right)
$$

by restriction to

$$
\bigcup_{b \in S_{k}} \pi_{E}^{-1}(b)
$$

followed by integration along the fibres of

$$
\pi_{D}: \bigcup_{b \in S_{k}} \pi_{E}^{-1}(b) \rightarrow \bigcup_{b \in S_{k}} M_{b}^{\prime} .
$$

We will see in the proof that it is this map which induces the isomorphism in the theorem.

Note that this theorem is a generalization of Sniatycki's result for reducible real polarization in [6].

Proof. The inclusion

$$
i: D \rightarrow \bar{P}
$$

induces a filtration on the complex (3), by letting

$$
F^{p}\left(\Lambda^{l} \bar{P}^{*} \otimes \mathscr{L}^{k} \otimes \delta_{P}\right)=\operatorname{Ann}\left(\Lambda^{l-p+1} D \wedge \Lambda^{p-1} \bar{P} \otimes \mathscr{L}^{k} \otimes \delta_{P}\right)
$$

and

$$
F^{p} C^{\infty}\left(\Lambda^{l} \bar{P}^{*} \otimes \mathscr{L}^{k} \otimes \delta_{P}\right)=C^{\infty}\left(F^{p}\left(\Lambda^{l} \bar{P}^{*} \otimes \mathscr{L}^{k} \otimes \delta_{P}\right)\right) .
$$

Associated to this filtered complex we have a spectral sequence $\left\{E_{r}\right\}$, where

$$
E_{0}^{p, q}=\frac{F^{p} C^{\infty}\left(\Lambda^{p+q} \bar{P}^{*} \otimes \mathscr{L}^{k} \otimes \delta_{P}\right)}{F^{p+1} C^{\infty}\left(\Lambda^{p+q} \bar{P}^{*} \otimes \mathscr{L}^{k} \otimes \delta_{P}\right)},
$$

and the differential is induced from $\nabla^{P}$.

Note that if $q>m$ then

$$
E_{0}^{p, q}=0,
$$

and if $p>n-m$ then

$$
E_{0}^{p, q}=0
$$


By general theory we have that the spectral sequence converges to

$$
E_{\infty}^{p, q}=\mathrm{Gr}^{p}\left(H^{p+q}\left(M, \nabla^{P}\right)\right) .
$$

We shall now compute this spectral sequence. Let $\varphi \in E_{0}^{p, q}$. We may then in the local coordinates $(x, y, z, \bar{z})$, constructed earlier in this section, represent

$$
\varphi=\left[\sum_{|I|=p} \eta_{I}(x, z, \bar{z}) \wedge d \bar{z}_{I}\right],
$$

where $\eta_{I}$ is a $q$-form on $T^{m}$ :

$$
\eta_{I}=\sum_{|J|=q} \eta_{I}^{J}(x, y, z, \bar{z}) d y_{J}
$$

The differential

$$
d_{0}: E_{0}^{p, q} \rightarrow E_{0}^{p, q+1}
$$

is then given by

$$
d_{0} \varphi=\left[\sum_{|I|=p}\left(d_{D}+i \beta\right) \eta_{I}(x, z, \bar{z}) \wedge d \bar{z}_{I}\right] .
$$

Hence we see that if $d_{0} \varphi=0$ in $E_{0}^{p, q+1}$, then

$$
\left(d_{D}+i \beta\right) \eta_{I}(x, z, \bar{z})=0 .
$$

By the expression for $\eta_{I}$ we get thus that

$$
\begin{aligned}
& \left(d_{D}+i \beta\right) \eta_{I}(x, z, \bar{z}) \\
& =\sum_{i=1}^{m} \sum_{|J|=q}\left(\frac{\partial \eta_{I}^{J}}{\partial y_{i}}(x, y, z, \bar{z})+i x_{i} \eta_{I}^{J}(x, y, z, \bar{z})\right) d y_{i} \wedge d y_{J} .
\end{aligned}
$$

Following Śniatycki's local analysis of the leafwise complex of a real polarization in [6], we argue as follows using Fourier series:

Write

$$
\eta_{I}^{J}(x, y, z, \bar{z})=\sum_{n \in \mathbf{Z}^{m}} \eta_{I}^{J}(n)(x, z, \bar{z}) \exp (-i\langle n, y\rangle) .
$$

Then (6) becomes

$$
\sum_{i=1}^{m}\left(x_{i}-n_{i}\right) d y_{i} \wedge\left(\sum_{|J|=q} \eta_{I}^{J}(n)(x, z, \bar{z}) d y_{J}\right)=0
$$

for all $n \in \mathbb{Z}^{m}$. Suppose now that $q<m$. It then follows by Lemma 3.7 in [6], that there exist $\psi_{I}^{J}(n)(x, z, \bar{z})$, for all $n \in \mathbb{Z}^{m}$, such that

$$
\sum_{i=1}^{m}\left(x_{i}-n_{i}\right) d y_{i} \wedge\left(\sum_{|J|=q-1} \psi_{I}^{J}(n)(x, z, \bar{z}) d y_{J}\right)=\eta_{I}^{J}(n)(x, z, \bar{z}),
$$


and so we can construct $\psi_{I}^{W} \in C^{\infty}\left(W, \Lambda^{q-1} D^{*}\right)$ by defining

$$
\psi_{I}^{W}=\sum_{|J|=q-1} \sum_{n \in \mathbf{Z}^{m}} \psi_{I}^{J}(n)(x, z, \bar{z}) \exp (-i<n, y>) d y_{J}
$$

By construction we have that

$$
\left(d_{D}+i \beta\right) \psi_{I}^{W}=\eta_{I}
$$

Choose a partition of unity $\left\{V \times U, \rho_{V \times U}\right\}$ on $M^{\prime}$. By pulling back to $M$, we get a partition of unity $\left\{W, \rho_{W}\right\}$ on $M$. Using this partition of unity, we can define $\psi \in E_{0}^{p, q}$ by

$$
\psi=\sum_{W} \rho_{W} \sum_{|I|=p} \psi_{I}^{W} \wedge d \bar{z}_{I}
$$

Then

$$
\begin{aligned}
d_{0} \psi & =\left[\nabla^{P} \sum_{W} \rho_{W} \sum_{|I|=p} \psi_{I}^{W} \wedge d \bar{z}_{I}\right] \\
& =\left[\sum_{W} \rho_{W} \sum_{|I|=p} \eta_{I} \wedge d \bar{z}_{I}\right] \\
& =\varphi .
\end{aligned}
$$

Hence we see

$$
E_{1}^{p, q}=0
$$

if $q<m$.

It is clear that

$$
E_{0}^{p, m}=\operatorname{ker} d_{0},
$$

so

We have a map

$$
E_{1}^{p, m}=\frac{E_{0}^{p, m}}{\operatorname{Im} d_{0}}
$$

$$
\left(\pi_{D}\right)_{*}: E_{0}^{p, m} \rightarrow \prod_{b \in S_{k}} C^{\infty}\left(M_{b}^{\prime}, \Omega^{0, p}\left(\mathscr{L}_{b}^{k} \otimes K_{b}^{\frac{1}{2}}\right)\right),
$$

given by

$$
\left(\pi_{D}\right)_{*}(\varphi)=\sum_{b \in S_{k}}\left(\pi_{D}\right)_{*} \varphi(b),
$$

where $\left(\pi_{D}\right)_{*} \varphi(b)$ is the integration along the fibres of the fibration $\pi_{D}: \pi_{E}^{-1}(b) \rightarrow$ $M_{b}^{\prime}$. Note that $\left(\pi_{D}\right)_{*}$ is in fact well-defined on $E_{1}^{p, m}$, so we get

$$
\left(\pi_{D}\right)_{*}: E_{1}^{p, m} \rightarrow \prod_{b \in S_{k}} C^{\infty}\left(M_{b}^{\prime}, \Omega^{0, p}\left(\mathscr{L}_{b}^{k} \otimes K_{b}^{\frac{1}{2}}\right)\right)
$$

In terms of the local Fourier series description of $\varphi$, we see that the condition $\left(\pi_{D}\right)_{*} \varphi=0$ implies that

$$
\eta_{I}^{J}(0)(0, z, \bar{z})=0 \text {. }
$$

That is precisely the condition one needs on $\eta_{I}$ in order to be able, locally in the coordinates $(x, y, z, \bar{z})$, to solve the following equation for $\psi_{I}^{J}(n)$,

$$
i \sum_{i=1}^{m}\left(x_{i}-n_{i}\right) d y_{i} \wedge\left(\sum_{|J|=m-1} \psi_{I}^{J}(n)(x, z, \bar{z}) d y_{J}\right)=\eta_{I}^{J}(n)(x, z, \bar{z}) d y_{J}
$$


By exactly the same argument as in the case where $q<m$, we see that using a partition of unity and these local solutions, we can construct $\psi \in E_{0}^{p, m-1}$ such that $d_{0} \psi=\varphi$. Hence the map $\left(\pi_{D}\right)_{*}$ is injective.

Using the partition of unity $\left\{W, \rho_{W}\right\}$ one easily constructs an $m$-form $\psi$ on $M$, such that

$$
\int_{\pi_{D}^{-1}\left(p^{\prime}\right)} \psi=1
$$

for any $p^{\prime} \in M_{b}^{\prime}, b \in S_{k}$. For

$$
\tilde{\varphi} \in \prod_{b \in S_{k}} C^{\infty}\left(M_{b}^{\prime}, \Omega^{p}\left(\mathscr{L}_{b}^{k} \otimes K_{b}^{\frac{1}{2}}\right)\right),
$$

we let

$$
\varphi=\left(\pi_{D}\right)^{*}(\tilde{\varphi}) \wedge \psi .
$$

Then $\varphi \in E_{0}^{p, m}$ and $\left(\pi_{D}\right)_{*} \varphi=\tilde{\varphi}$. We thus see that (8) is an isomorphism.

Consider the map

$$
d_{1}: E_{1}^{p, m} \rightarrow E_{1}^{p+1, m}
$$

given by

$$
d_{1} \varphi=\left[\sum_{|I|=p} \bar{\partial}_{x} \eta_{I}(x, y, z, \bar{z}) d \bar{z}_{I}\right] .
$$

It is clear from the definition that

$$
\left(\pi_{D}\right)_{*} \circ d_{1}=\bar{\partial}_{b} \circ\left(\pi_{D}\right)_{*} .
$$

Hence $\left(\pi_{D}\right)_{*}$ induces the following isomorphism on cohomology:

$$
E_{2}^{p, m} \cong \prod_{b \in S_{k}} H^{p}\left(M_{b}^{\prime}, \bar{\partial}_{b}\right)
$$

Hence we see that the spectral sequence degenerates at the $E_{2}$-term, and the general theory gives

$$
E_{2}^{p, q}=\frac{F^{p} H^{p+q}\left(M, \nabla^{P}\right)}{F^{p+1} H^{p+q}\left(M, \nabla^{P}\right)} .
$$

By a standard derivation this result implies the result stated in the theorem.

\section{A Lefschetz Fixed Point Theorem for Reducible Non-Negative Polarizations}

We shall make the same assumption on the symplectic manifold $(M, \omega)$ and the polarization $P$ as in Sect. 2. Moreover, we will for the rest of the paper assume that $M$ is compact.

Suppose we have a symplectomorphism $f$ of the symplectic manifold $M$. Assume we are given a lift of $f$ to the line bundle $\mathscr{L}$. We shall require that $f$ preserves $P$ and that we are given a lift of $f$ to the line bundle $\delta_{P}$. Then $f$ induces an endomorphism of the cohomology groups of the complex (3).

Since $f$ preserves $P$, it also preserves $E$ and $D$, and hence induces a map on the leafspaces $M / E=B$ and $M / D=M^{\prime}$. We will denote these maps $f_{B}: B \rightarrow B$ and $f^{\prime}: M^{\prime} \rightarrow M^{\prime}$. It is clear that $f_{b}^{\prime}: M_{b}^{\prime} \rightarrow M_{f_{B}(b)}^{\prime}$ will be holomorphic with respect 
to the Kähler structure induced on $M_{b}^{\prime}$ by $P$. Suppose $b \in B$ is a fixed point for $f_{B}: B \rightarrow B$. Let $p^{\prime} \in \pi_{B}^{-1}(b)$. We shall now study $f$ in the local coordinates on $M$ around $\pi_{D}^{-1}\left(p^{\prime}\right)$ we constructed in Sect. 2 . In these coordinates we can write

$$
f(x, y, z, \bar{z})=\left(f_{1}(x, y, z, \bar{z}), f_{2}(x, z, \bar{z})(y), f_{3}(x, y, z, \bar{z}), \overline{f_{3}}(x, y, z, \bar{z})\right),
$$

where

$$
f_{2}(x, z, \bar{z}): T^{m} \rightarrow T^{m}
$$

We calculate that

$$
\begin{aligned}
& d f\left(\frac{\partial}{\partial y_{i}}\right)=\sum_{j} \frac{\partial f_{1}^{j}}{\partial y_{i}} \frac{\partial}{\partial x_{j}}+\sum_{j} \frac{\partial f_{2}^{j}}{\partial y_{i}} \frac{\partial}{\partial y_{j}}+\sum_{j} \frac{\partial f_{3}^{j}}{\partial y_{i}} \frac{\partial}{\partial z_{j}}+\sum_{j} \frac{\partial \bar{f}_{3}^{j}}{\partial y_{i}} \frac{\partial}{\partial \bar{z}_{j}}, \\
& d f\left(\frac{\partial}{\partial z_{i}}\right)=\sum_{j} \frac{\partial f_{1}^{j}}{\partial z_{i}} \frac{\partial}{\partial x_{j}}+\sum_{j} \frac{\partial f_{2}^{j}}{\partial z_{i}} \frac{\partial}{\partial y_{j}}+\sum_{j} \frac{\partial f_{3}^{j}}{\partial z_{i}} \frac{\partial}{\partial z_{j}}+\sum_{j} \frac{\partial \bar{f}_{3}^{j}}{\partial z_{i}} \frac{\partial}{\partial \bar{z}_{j}}, \\
& d f\left(\frac{\partial}{\partial \bar{z}_{i}}\right)=\sum_{j} \frac{\partial f_{1}^{j}}{\partial \bar{z}_{i}} \frac{\partial}{\partial x_{j}}+\sum_{j} \frac{\partial f_{2}^{j}}{\partial \bar{z}_{i}} \frac{\partial}{\partial y_{j}}+\sum_{j} \frac{\partial f_{3}^{j}}{\partial \bar{z}_{i}} \frac{\partial}{\partial z_{j}}+\sum_{j} \frac{\partial \bar{f}_{3}^{j}}{\partial \bar{z}_{i}} \frac{\partial}{\partial \bar{z}_{j}} .
\end{aligned}
$$

Since $f$ preserves $P, E$ and $D$, we can in particular conclude that

$$
\begin{aligned}
\frac{\partial f_{1}^{j}}{\partial y_{i}}=\frac{\partial f_{1}^{j}}{\partial z_{i}} & =\frac{\partial f_{1}^{j}}{\partial \bar{z}_{i}}=0, \\
\frac{\partial f_{3}^{j}}{\partial y_{i}} & =0 .
\end{aligned}
$$

The symplectic form $\omega$ is also preserved by $f$, so we get from (4) that

$$
\begin{aligned}
f^{*} \omega= & \sum_{i=1}^{m} d f_{1}^{i} \wedge d f_{2}^{i}+\sum_{i=m+1}^{n} f^{*}\left(A_{i j}+\frac{\partial^{2} K}{\partial x_{i} \partial \bar{z}_{j}}\right) d f_{1}^{i} \wedge d \bar{f}_{3}^{j} \\
& +\sum_{i=m+1}^{n} f^{*} B_{i j} d f_{1}^{i} \wedge d f_{3}^{j}+\sum_{i=m+1}^{n} f^{*} \frac{\partial^{2} K}{\partial z_{i} \partial \bar{z}_{j}} d f_{3}^{i} \wedge d \bar{f}_{3}^{j} \\
= & \omega .
\end{aligned}
$$

From this it follows that

$$
\sum_{i=1}^{n} \frac{\partial f_{1}^{i}}{\partial x_{j}} \frac{\partial f_{2}^{i}}{\partial y_{k}}=\delta_{j k}
$$

We have the following identifications:

$$
\begin{aligned}
& \left(d_{B} f\right)_{i j}=\frac{\partial f_{1}^{i}}{\partial x_{j}}, \\
& \left(d_{D} f\right)_{i j}=\frac{\partial f_{2}^{i}}{\partial y_{j}},
\end{aligned}
$$

so (9) is just saying

$$
\left(d_{B} f\right)^{t} d_{D} f=1
$$


From this it follows that

$$
\frac{\partial^{2} f_{2}^{i}}{\partial y_{k} \partial y_{j}}=0, \quad k, j=1, \ldots, m,
$$

so we see that there exists $\Delta \in G L(m, \mathbb{Z})$ and a smooth map $c: V \times U \rightarrow \mathbb{R}^{m}$, such that

$$
f_{2}^{i}(x, z, \bar{z})(y)=\sum_{j=1}^{m} \Delta_{i j} y_{j}+c_{i}(x, z, \bar{z}),
$$

where $\Delta=d_{D} f$. Moreover, since

$$
\operatorname{Det}\left(d_{B} f\right) \operatorname{Det}\left(d_{D} f\right)=1 \text {, }
$$

we conclude that $\operatorname{Det}\left(d_{D} f\right)(p), p \in M$, only depends on $\pi_{E}(p) \in B$.

The following proposition computes the trace on the cohomology groups of any symplectomorphism $f$ that preserves $P$ on the basis of the local description of $f$.

Proposition 3.1. Let $P$ be a reducible non-negative polarization on a compact symplectic manifold. Suppose $f$ is a symplectomorphism of $M$ that preserves $P$. Assume moreover we have a lift $f^{*}$ of $f$ to $\mathscr{L} \otimes \delta_{P}$. Then

$$
\begin{aligned}
& \sum_{i=0}^{n}(-1)^{i} \operatorname{Tr}\left(f^{*}: H^{n}\left(M, \nabla^{P}\right)\right) \\
& \quad=(-1)^{m} \sum_{\left\{b \in S_{k} \mid f_{B}(b)=b\right\}} \operatorname{Det}\left(d_{D} f\right)(b) \sum_{i=0}^{n-m}(-1)^{i} \operatorname{Tr}\left(f_{b}^{*}: H^{i}\left(M_{b}^{\prime}, \bar{\partial}_{b}\right)\right),
\end{aligned}
$$

where $f_{b}^{\prime *}$ is the lift of $f_{b}^{\prime}$ to $\mathscr{L}_{b}^{k} \otimes K_{b}^{\frac{1}{2}}$, constructed from $\left.f^{*}\right|_{\pi_{E}^{-1}\left(\left\{b \in S_{k} \mid f_{B}(b)=b\right\}\right)}$ the lift of $\left.f\right|_{\pi_{E}^{-1}\left(\left\{b \in S_{k} \mid f_{B}(b)=b\right\}\right)}$ to $\left.\mathscr{L}^{k} \otimes \delta_{P}\right|_{\pi_{E}^{-1}\left(\left\{b \in S_{k} \mid f_{B}(b)=b\right\}\right)}$.

Note that there is no assumption on the nature of the fixed point set of $f$ in this proposition.

Proof. Recall the spectral sequence from the proof of Theorem 2.1. We have that the following diagram is commutative

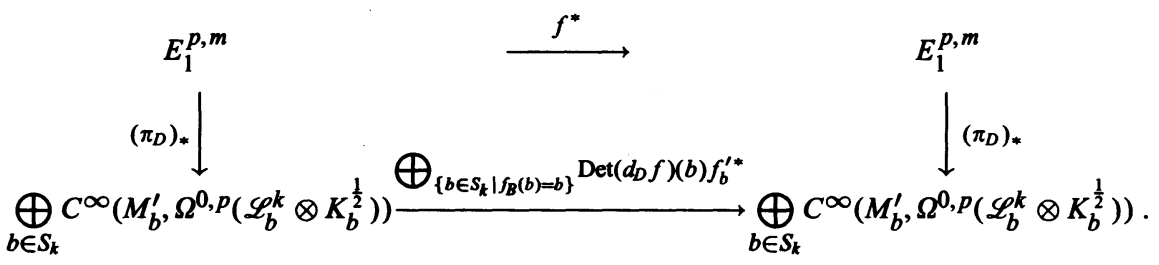

Hence we get

$$
\operatorname{Tr}\left(f^{*}: E_{2}^{p, m} \rightarrow E_{2}^{p, m}\right)=\sum_{\left\{b \in S_{k} \mid f_{B}(b)=b\right\}} \operatorname{Det}\left(d_{D} f\right)(b) \operatorname{Tr}\left(f_{b}^{\prime *}: H^{p}\left(M_{b}^{\prime}, \bar{\partial}_{b}\right)\right) .
$$

The result follows from this equality.

Using Proposition 3.1, we can now give a fixed point formula for the trace of the action of $f$ on the cohomology groups, in the case where $f$ has simple fixed points. 
Theorem 3.1. Assume $P$ is a reducible non-negative polarization on a compact symplectic manifold. Suppose $f$ is a symplectomorphism of $M$ that preserves $P$. We shall assume that $f$ has simple fixed points. Assume moreover that we have a lift $f^{*}$ of $f$ to $\mathscr{L} \otimes \delta_{P}$. Then

$$
\begin{aligned}
& \sum_{i=0}^{n}(-1)^{i} \operatorname{Tr}\left(f^{*}: H^{i}\left(M, \nabla^{P}\right)\right) \\
& \quad=\sum_{\{p \in M \mid f(p)=p\}} v_{p} \sum_{i=0}^{n}(-1)^{i} \frac{\operatorname{Tr}\left(f_{p}^{*}: \Lambda^{i} \bar{P}_{p}^{*} \otimes \mathscr{L}_{p}^{k} \otimes \delta_{P_{p}}\right)}{|\operatorname{Det}(1-d f(p))|},
\end{aligned}
$$

where

$$
v_{p}=\operatorname{SignDet}\left(1-d f_{B}(p)\right) \text {. }
$$

Before proving this theorem, we shall compute the number of fixed points for an affine diffeomorphism of an $\boldsymbol{m}$-dimensional torus.

Let $\Delta \in G L(m, \mathbb{Z})$ and $y_{0} \in \mathbb{R}^{m}$. Consider the map $F: \mathbb{R}^{m} \rightarrow \mathbb{R}^{m}$ given by

$$
F(y)=\Delta(y)+y_{0}
$$

This map induces a diffeomorphism of the torus. We will also call this diffeomorphism F.

Lemma 3.1. Suppose $\operatorname{Det}(\Delta-1) \neq 0$. Then $F: T^{m} \rightarrow T^{m}$ has finitely many fixed points and the number of fixed points is $|\operatorname{Det}(\Delta-1)|$.

Proof. We calculate

$$
\begin{gathered}
F(y)=y \Leftrightarrow \\
\exists \lambda \in \mathbb{Z}^{m}:(\Delta-1) y=y_{0}+\lambda .
\end{gathered}
$$

So we see that the number of fixed points is finite and it is given by the order of

$$
\frac{(\Delta-1)^{-1} \mathbb{Z}^{m}}{\mathbb{Z}^{m}},
$$

which is the same as $\left|\mathbb{Z}^{m} /(\Delta-1) \mathbb{Z}^{m}\right|$. It is a simple exercise to prove that

$$
\left|\frac{\mathbb{Z}^{m}}{(\Delta-1) \mathbb{Z}^{m}}\right|=|\operatorname{Det}(\Delta-1)| \text {. }
$$

Using Proposition 3.1 and Lemma 3.1 it is easy to prove Theorem 3.1

Proof of Theorem 3.1. By Proposition 3.1 and the holomorphic Lefschetz fixed point theorem we get

$$
\begin{aligned}
& \sum_{i=0}^{n}(-1)^{i} \operatorname{Tr}\left(f^{*}: H^{i}\left(M, \nabla^{P}\right)\right) \\
& =(-1)^{m} \sum_{\left\{b \in S_{k} \mid f_{B}(b)=b\right\}} \operatorname{Det}\left(d_{D} f\right)(b) \\
& \quad \times \sum_{\left\{p \in M_{b}^{\prime} \mid f_{b}^{\prime}(p)=p\right\}} \sum_{i=0}^{n-m}(-1)^{i} \frac{\operatorname{Tr}\left(f_{b}^{\prime *}:\left(\Lambda^{0, i} M_{b}^{\prime}\right)_{p} \otimes\left(\mathscr{L}_{b}^{k} \otimes \delta_{P_{b}}\right)_{p}\right)}{\left|\operatorname{Det}\left(1-d f_{b}^{\prime}(p)\right)\right|} .
\end{aligned}
$$


Using Lemma 3.1, we can write this as

$$
\begin{aligned}
\sum_{i=0}^{n}(-1)^{i} \operatorname{Tr}\left(f^{*}: H^{i}\left(M, \nabla^{P}\right)\right) \\
=(-1)^{m} \sum_{\left\{b \in S_{k} \mid f_{B}(b)=b\right\}} \frac{\operatorname{Det}\left(d_{D} f\right)(b)}{\left|\operatorname{Det}\left(1-d_{D} f\right)(b)\right|} \sum_{\left\{p \in \pi_{E}^{-1}(b) \mid f(p)=p\right\}} \\
\quad \times \sum_{i=0}^{n-m}(-1)^{i} \frac{\operatorname{Tr}\left(f_{b}^{\prime *}:\left(\Lambda^{0, i} M_{b}^{\prime}\right)_{\pi_{D}(p)} \otimes\left(\mathscr{L}_{b}^{k} \otimes \delta_{P_{b}}\right)_{\pi_{D}(p)}\right)}{\left|\operatorname{Det}\left(1-d f_{b}^{\prime}(p)\right)\right|} .
\end{aligned}
$$

We note that if $b \in B$ is a fixed point for $f_{B}$, but $\mathrm{b}$ is not a Bohr-Sommerfeld leaf, then

$$
\sum_{\left\{p \in \pi_{E}^{-1}(b) \mid f(p)=p\right\}} \sum_{i=0}^{n-m}(-1)^{i} \frac{\operatorname{Tr}\left(f_{b}^{\prime *}:\left(\Lambda^{0, i} M_{b}^{\prime}\right)_{\pi_{D}(p)} \otimes\left(\mathscr{L}_{b}^{k} \otimes \delta_{P_{b}}\right)_{\pi_{D}(p)}\right)}{\left|\operatorname{Det}\left(1-d f_{b}^{\prime}(p)\right)\right|}=0 .
$$

This follows by applying the usual Lefschetz fixed point theorem to the elliptic complex

$$
0 \rightarrow C^{\infty}\left(\pi_{E}^{-1}(b), \mathscr{L}^{k} \otimes \delta_{P}\right) \stackrel{\nabla^{P}}{\rightarrow} \cdots \stackrel{\nabla^{P}}{\rightarrow} C^{\infty}\left(\pi_{E}^{-1}(b), \Lambda^{n} \bar{P}^{*} \otimes \mathscr{L}^{k} \otimes \delta_{P}\right) \rightarrow 0
$$

and the fact that the cohomology groups of this complex are all trivial. Combining this with the previous result, we get

$$
\begin{aligned}
\sum_{i=0}^{n}( & -1)^{i} \operatorname{Tr}\left(f^{*}: H^{i}\left(M, \nabla^{P}\right)\right) \\
= & (-1)^{m} \sum_{\{p \in M \mid f(p)=p\}} \frac{\operatorname{Det}\left(d_{D} f\right)(p)}{\left|\operatorname{Det}\left(1-d_{D} f\right)(p)\right|} \\
& \times \sum_{i=0}^{n-m}(-1)^{i} \frac{\operatorname{Tr}\left(f_{b}^{\prime *}:\left(\Lambda^{0, i} M_{b}^{\prime}\right)_{\pi_{D}(p)} \otimes\left(\mathscr{L}_{b}^{k} \otimes \delta_{P_{b}}\right)_{\pi_{D}(p)}\right)}{\left|\operatorname{Det}\left(1-d f_{b}^{\prime}(p)\right)\right|} .
\end{aligned}
$$

From (9) we get that

$$
\begin{aligned}
\operatorname{Det}\left(\left(1-d f_{B}\right)\left(d_{D} f\right)^{t}\right) & =\operatorname{Det}\left(d_{D} f-1\right) \\
& =(-1)^{m} \operatorname{Det}\left(1-d_{D} f\right) .
\end{aligned}
$$

Since

$$
\operatorname{Det}\left(1-d_{D} f\right)=\sum_{i=0}^{m}(-1)^{i} \operatorname{Tr}\left(\Lambda^{i}\left(d_{D} f\right)\right)
$$

we get that

$$
\begin{aligned}
& \sum_{i=0}^{n}(-1)^{i} \operatorname{Tr}\left(f^{*}: H^{i}\left(M, \nabla^{P}\right)\right) \\
& \quad=\sum_{\{p \in M \mid f(p)=p\}} v_{p} \sum_{i=0}^{n}(-1)^{i} \frac{\operatorname{Tr}\left(f^{*}(p): \Lambda^{i} \bar{P}_{p}^{*} \otimes \mathscr{L}_{p}^{k} \otimes \delta_{P_{p}}\right)}{|\operatorname{Det}(1-d f(p))|},
\end{aligned}
$$

where

$$
v_{p}=\operatorname{Sign} \operatorname{Det}\left(1-d f_{B}(p)\right)
$$


Here we have used that

$$
|\operatorname{Det}(1-d f(p))|=\left|\operatorname{Det}\left(1-d f_{B}(p)\right)\right|\left|\operatorname{Det}\left(1-d f_{D}(p)\right) \| \operatorname{Det}\left(1-d f_{b}^{\prime}(p)\right)\right|,
$$

but this follows from the fact that $f$ preserves $D$ and $E$.

\section{An Index Theorem for Reducible Non-Negative Polarizations}

Suppose $M$ is a compact symplectic manifold and assume that $P^{\prime}$ is a Kähler polarization on $M$. We can then identify $\delta_{P^{\prime}}=K^{\frac{1}{2}}$, where $K$ is the canonical bundle. The complex (3) for $P^{\prime}$ becomes the $\bar{\partial}$-complex with values in $\mathscr{L}^{k} \otimes K^{\frac{1}{2}}$, which is elliptic, and by the Hirzebruch-Riemann-Roch formula we get

$$
\begin{aligned}
\operatorname{Index}\left(\nabla^{P^{\prime}}\right) & =\int_{M} \operatorname{ch}\left(\mathscr{L}^{k} \otimes K^{\frac{1}{2}}\right) \wedge \operatorname{ch}\left(K^{-\frac{1}{2}}\right) \wedge \widehat{A}(T M) \\
& =\int_{M} \operatorname{ch}\left(\mathscr{L}^{k}\right) \wedge \widehat{A}(T M) .
\end{aligned}
$$

This formula shows that Index $\left(\nabla^{P^{\prime}}\right)$ for Kähler polarizations $P^{\prime}$ on $M$ only depends on the symplectic structure of $M$, since $\operatorname{ch}\left(\mathscr{L}^{k}\right)=\exp (k \omega)$.

We shall now prove the following analogue of the Hirzebruch-Riemann-Roch index theorem for reducible non-negative polarizations $P$, which satisfies the following somewhat technical assumption. We shall assume that the $T^{m}$ bundle $\pi_{D}: M \rightarrow M^{\prime}$ admits a flat connection. Note that with this connection, we can define a closed 2 -form $\omega_{1}$ on $M$ which in the local symplectic coordinates $(x, y, z, \bar{z})$ constructed in Sect. 2 is given by

$$
\omega_{1}=\sum_{i=1}^{m} d x_{i} \wedge d y_{i} .
$$

Notice that this condition is trivially satisfied for real polarizations and Kähler polarizations.

Theorem 4.1. Let $M$ be a compact symplectic manifold. Suppose we have a reducible non-negative polarization $P$ on $M$ such that the $T^{m}$ bundle $\pi_{D}: M \rightarrow M^{\prime}$ admits a flat connection. The index of the complex (3) is given by

$$
\operatorname{Index}\left(\nabla^{P}\right)=(-1)^{m} \int_{M} \operatorname{ch}\left(\mathscr{L}^{k}\right) \wedge \widehat{A}(T M) .
$$

From the proof it will follow that if $P$ is a real polarization, then the right-hand side of (11) just reduces to the volume of $(M, k \omega)$. So in short the theorem is in that case saying that the cardinality of the level $k$ Bohr-Sommerfeld set is given by the volume of $(M, k \omega)$ :

Corollary 4.1. Let $M$ be a compact symplectic manifold with a reducible real polarization $P$ and a prequantum line bundle $(\mathscr{L}, \nabla)$. Let $S_{k}$ be the level $k$ BohrSommerfeld set for $P$ and $(\mathscr{L}, \nabla)$ as defined in Definition 2.3. Then

$$
\# S_{k}=k^{n} \operatorname{Vol}(\mathrm{M}) \text {. }
$$


Proof of Theorem 4.1. First of all we notice that using the symplectic form, we get an isomorphism

$$
\begin{aligned}
T M & \cong D \oplus \pi_{D}^{*} T M^{\prime} \\
& \cong \pi_{E}^{*} T^{*} B \oplus \pi_{D}^{*} T M^{\prime} .
\end{aligned}
$$

Recall from Sect. 2 that we constructed a lattice inside $\pi_{B}^{*}\left(T^{*} B\right)$, hence the bundle $\pi_{B}^{*}\left(T^{*} B\right)$ is flat. This means that

$$
\hat{A}(T M)=\pi_{D}^{*}\left(\hat{A}\left(T M^{\prime}\right)\right) .
$$

The left-hand side of (11) becomes

$$
\begin{aligned}
\int_{M} \exp (k \omega) \wedge \hat{A}(T M) & =\int_{B}\left(\pi_{E}\right)_{*}(\exp (k \omega) \wedge \hat{A}(T M)) \\
& =\int_{B}\left(\pi_{B}\right)_{*}\left(\left(\pi_{D}\right)_{*}(\exp (k \omega)) \wedge \hat{A}\left(T M^{\prime}\right)\right) .
\end{aligned}
$$

Using the local expression (4) for the symplectic form we have that

$$
\omega=\omega_{1}+\omega_{2}+\omega_{3},
$$

where

$$
\begin{gathered}
\omega_{1}=\sum_{i=1}^{m} d x_{i} \wedge d y_{i}, \\
\omega_{2}=\sum_{i=m+1}^{n}\left(A_{i j}+\frac{\partial^{2} K}{\partial x_{i} \partial \bar{z}_{j}}\right) d x_{i} \wedge d \bar{z}_{j}+\sum_{i=m+1}^{n} B_{i j} d x_{i} \wedge d z_{j}, \\
\omega_{3}=\sum_{i=m+1}^{n} \frac{\partial^{2} K}{\partial z_{i} \partial \bar{z}_{j}} d z_{i} \wedge d \bar{z}_{j} .
\end{gathered}
$$

Here $\omega_{3}$ is a local expression for $\omega_{b}$, the reduction of $\omega$ to $M_{b}^{\prime}$.

We then see that

$$
\begin{aligned}
\left(\pi_{D}\right)_{*}(\exp (k \omega)) & =\left(\pi_{D}\right)_{*}\left(\sum_{i=0}^{n} \frac{1}{i !} \sum_{j=0}^{i}\left(\begin{array}{l}
i \\
j
\end{array}\right)\left(k \omega_{1}\right)^{j}\left(k\left(\omega_{2}+\omega_{3}\right)\right)^{i-j}\right) \\
& =k^{m} d x_{1} \wedge \cdots \wedge d x_{m} \sum_{i=m}^{n} \frac{1}{(i-m) !}\left(k\left(\omega-\omega_{1}\right)\right)^{i-m}
\end{aligned}
$$

Note that $\omega-\omega_{1}$ is closed and basic with respect to $\pi_{D}$, hence it is the pull back of a form on $M^{\prime}$. We shall also denote that form by $\omega-\omega_{1}$. Let $\Lambda$ be the submanifold of $\pi_{B}^{*}\left(T^{*} B\right)$, which consists of points in $\pi_{B}^{*}\left(T^{*} B\right)$, that acts trivially on the corresponding fibres. We will use the notation $p$ for the projection $p: \pi_{B}^{*}\left(T^{*} B\right) / \Lambda \rightarrow M^{\prime}$.

Let us now define a section $s_{k}: M^{\prime} \rightarrow \pi_{B}^{*}(T B) / \Lambda^{*}$ by the following requirement. Note that any $\alpha_{p^{\prime}} \in \Lambda_{p^{\prime}}$ represents a homology class in $H_{1}\left(\pi_{D}^{-1}\left(p^{\prime}\right), \mathbb{Z}\right)$, for $p^{\prime} \in$ $\pi_{B}^{-1}(b)$. We then define $s_{k}\left(p^{\prime}\right) \in \pi_{B}^{*}\left(T_{b} B\right) / \Lambda_{p^{\prime}}^{*}$ by

$$
\exp \left(2 \pi i \alpha_{p^{\prime}}\left(s_{k}\left(p^{\prime}\right)\right)\right)=\operatorname{Hol}_{\left[\alpha_{p^{\prime}}\right]}\left(\left.\mathscr{L}^{k} \otimes \delta_{P}\right|_{\pi_{D}^{-1}\left(p^{\prime}\right)}\right),
$$

for $p^{\prime} \in \pi_{B}^{-1}(b)$. Here $\operatorname{Hol}_{\left[\alpha_{p^{\prime}}\right]}\left(\left.\mathscr{L}^{k} \otimes \delta_{P}\right|_{\pi_{D}^{-1}\left(p^{\prime}\right)}\right)$ is the holonomy of the flat bundle $\left.\mathscr{L}^{k} \otimes \delta_{P}\right|_{\pi_{D}^{-1}\left(p^{\prime}\right)}$ around $\left[\alpha_{p^{\prime}}\right]$. Let $s_{0}: M^{\prime} \rightarrow \pi_{B}^{*}(T B) / \Lambda$ be the zero-section. 
We now note that

$$
\pi_{B}\left(p\left(s_{0}(B) \cap s_{k}(B)\right)\right)=S_{k}, \quad k=1,2, \ldots .
$$

Let us assume that we have chosen an orientation on $B$. If $B$ is not orientable, we will just lift the whole situation to the orientation cover of $B$. Since $M_{b}^{\prime}$ is oriented, we get an orientation on $M^{\prime}$. From the local study of the polarization we know there exist coordinates around each point $p^{\prime} \in M^{\prime}$, such that $s_{k}$ is given by

$$
s_{k}(x, z, \bar{z})=\sum_{i=1}^{n}\left(k x_{i}+x_{i}^{0}\right) \frac{\partial}{\partial x_{i}} .
$$

We can assume that $(x, z, \bar{z})$ are positive coordinates on $M^{\prime}$. The orientation of $M^{\prime}$ gives a canonical orientation of $\pi_{B}^{*} T B$. From the coordinate expression of $s_{k}$ we see that it is possible to orient $s_{k}\left(M^{\prime}\right)$, such that $s_{k}\left(M^{\prime}\right)$ and $s_{0}\left(M^{\prime}\right)$ always intersect positively in $\pi_{B}^{*}(T B) / \Lambda^{*}$. Now define a smooth $n$-form $\alpha$ on $\pi_{B}^{*}(T B) / \Lambda^{*}$ by

$$
\alpha_{b}=e_{1} \wedge \cdots \wedge e_{n},
$$

where $\left(e_{1}, \ldots, e_{n}\right)$ is a positive basis of $\Lambda_{p^{\prime}} \subset \pi_{B}^{*}\left(T_{\pi_{B}\left(p^{\prime}\right)}^{*} B\right)$. It is clear that $\alpha$ is the Poincaré dual of the zero section of $\pi_{B}^{*}(T B) / \Lambda^{*}$. Suppose we have a closed $2(n-m)$-form $\beta$ on $M^{\prime}$. We then get by Poincaré duality, that

$$
\begin{aligned}
\int_{p\left(s_{k}\left(M^{\prime}\right) \cap s_{0}\left(M^{\prime}\right)\right)} \beta & =\int_{s_{k}\left(M^{\prime}\right) \cap s_{0}\left(M^{\prime}\right)} p^{*}(\beta) \\
& =\int_{s_{k}\left(M^{\prime}\right)} \alpha \wedge p^{*}(\beta) \\
& =\int_{M^{\prime}} s_{k}^{*}\left(\alpha \wedge p^{*}(\beta)\right) \\
& =\int_{M^{\prime}} s_{k}^{*}(\alpha) \wedge \beta .
\end{aligned}
$$

The local expression for $s_{k}$ shows that

$$
s_{k}^{*}(\alpha)=k^{m} d x_{1} \wedge \cdots \wedge d x_{m} .
$$

Let $\beta$ be the closed form, which in local coordinates is given by

$$
\beta=\sum_{i=m}^{n} \frac{1}{(i-m) !}\left(k\left(\omega-\omega_{1}\right)\right)^{i-m} \wedge \hat{A}\left(T M^{\prime}\right) .
$$

By the previous calculations we get that

$$
\begin{aligned}
\int_{M} \exp (k \omega) \wedge \hat{A}(T M) & =\int_{p\left(s_{k}\left(M^{\prime}\right) \cap s_{0}\left(M^{\prime}\right)\right)} \sum_{i=m}^{n} \frac{1}{(i-m) !}\left(k\left(\omega-\omega_{1}\right)\right)^{i-m} \wedge \hat{A}\left(T M^{\prime}\right) \\
& =\sum_{p \in S_{k}} \int_{M_{b}^{\prime}} \sum_{i=0}^{n-m} \frac{1}{i !}\left(k \omega_{b}\right)^{i} \wedge \hat{A}\left(T M_{b}^{\prime}\right) .
\end{aligned}
$$

Here we have used that

$$
\hat{A}\left(i^{*}\left(T M^{\prime}\right)\right)=\hat{A}\left(T M_{b}^{\prime}\right),
$$

for the inclusion $i: M_{b}^{\prime} \rightarrow M^{\prime}$. 
By Theorem 2.1 and the Hirzebruch-Riemann-Roch theorem applied to $\left(M_{b}^{\prime}, \bar{\partial}_{b}\right)$, we thus get

$$
\operatorname{Index}\left(\nabla^{P}\right)=(-1)^{m} \int_{M} \operatorname{ch}\left(\mathscr{L}^{k}\right) \wedge \widehat{A}(T M) .
$$

In connection with this theorem we should mention that Guillemin and Sternberg in [4] have worked out explicitly the quantization of coadjoint orbits of $U(n)$ with respect to some non-trivial (singular) real polarizations. They find that the quantization agrees with the usual Kähler quantization.

\section{References}

1. Griffiths, P., Harris, J.: Principles of Algebraic Geometry. A Wiley-Interscience publication, New York: John Wiley \& Sons, 1978

2. Guillemin, V., and Sternberg, S.: Geometric asymptotics. Mathematical surveys Vol. 14, Providence, R.I.: Am. Math. Soc., 1977

3. Guillemin, V., Sternberg, S.: Symplectic techniques in Physics. Cambridge: Cambridge University Press, 1984

4. Guillemin, V., Sternberg, S.: The Gelfand-Cetlin System and quantization of the Complex Flag Manifold. J. Funct. Analysis 52, 106-128 (1983)

5. Kostant, B.: Orbits, Symplectic Structures and Representation Theory. Proceedings of the United States-Japan seminar in differential geometry, Kyoto, Japan, 1965, p. 71

6. Śniatycki, J.: On cohomology groups appearing in geometric quantization. Lecture Notes in Math. 570, Berlin: Springer-Verlag, 1977, pp. 46-66

7. Weinstein, A.: Lectures on symplectic manifolds, Conference Board of the Mathematical Sciences Regional Conference Series in Mathematics, 29

8. Woodhouse, N.: Geometric Quantization. Oxford: Oxford University Press, 1980 
